\title{
Mixed States of Composite Fermions Carrying Two and Four Vortices
}

\author{
K. Park ${ }^{\dagger}$ and J. K. Jain \\ Department of Physics, 104 Davey Laboratory, The Pennsylvania State University, Pennsylvania 16802
}

(November 1, 2018)

\begin{abstract}
There now exists preliminary experimental evidence for some fractions, such as $\nu=4 / 11$ and $5 / 13$, that do not belong to any of the sequences $\nu=n /(2 p n \pm 1), p$ and $n$ being integers. We propose that these states are mixed states of composite fermions of different flavors, for example, composite fermions carrying two and four vortices. We also obtain an estimate of the lowestexcitation dispersion curve as well as the transport gap; the gaps for 4/11 are smaller than those for $1 / 3$ by approximately a factor of 50 .

PACS numbers:71.10.Pm.
\end{abstract}

Two-dimensional electron systems exhibit spectacular phenomena when subjected to an intense, perpendicular magnetic field. Most remarkable is the fractional quantum Hall effect (FQHE) [1], in which the Hall resistance forms quantized plateaus at values $R_{H}=h / f e^{2}$ where $f$ is a simple rational fraction. The prominent fractions appear according to the primary sequences,

$$
f=\frac{n}{2 p n \pm 1}
$$

where $p$ and $n$ are integers. (The fractions $1-f$ are related to these by particle-hole symmetry.) An explanation of these sequences was one of the important initial successes of the composite fermion (CF) theory; it was in fact the clue that led to composite fermions [2 4]. A composite fermion is the bound state of an electron and an even number of quantum mechanical vortices of the many body wave function (sometimes thought of as an electron carrying an even number of magnetic flux quanta, where a flux quantum is defined as $\phi_{0}=h c / e$ ). The interacting electrons at Landau level (LL) filling factor $\nu=\nu^{*} /\left(2 p \nu^{*} \pm 1\right)$ transform into weakly interacting composite fermions with vorticity $2 p$ (denoted below by ${ }^{2 p} \mathrm{CFs}$ ) at an effective filling $\nu^{*}$. The integral quantum Hall effect of composite fermions, corresponding to $\nu^{*}=n$, manifests itself as the FQHE of electrons at $f=\frac{n}{2 p n \pm 1}$. These states are "pure", in the sense that they contain only a single flavor of composite fermions, namely ${ }^{2 p} \mathrm{CFs}$.

However, there now exist exceptions to the primary states. The FQHE at $\nu=5 / 2$ [5, 6] has been known for many years. There is growing consensus that its physical origin, while still formulated in terms of composite fermions, is fundamentally distinct from the other, odddenominator fractions: the $5 / 2$ state is described in terms of a BCS-type paired state of composite fermions [7], arising because the residual interaction between composite fermions is weakly attractive here [8], in contrast to the other fractions that are described as states containing an integral number of filled CF-LLs. The focus of this article will be on $\nu=4 / 11$ [9]. We suggest that it has a more or less the traditional description in terms of filled CFLLs, except that it is a "mixed" FQHE state of composite fermions of two different flavors, those carrying two and four vortices. (Here, the term "mixed" refers to an admixture of two different CF flavors, without necessarily implying spatial phase separation.)

Let us first see how the state at $\nu=4 / 11$ is understood in terms of a mixture of two different flavors of composite fermions. Start by considering the state of fully polarized electrons at $\nu=4 / 3$. The state at $4 / 3=1+1 / 3$ is incompressible, at least for certain class of interactions. It contains one fully occupied Landau level of electrons and the second Landau level at 1/3 filling. The electrons in the second Landau level are equivalent to composite fermions at effective filling of unity. Thus, the $4 / 3$ state is the simplest, albeit somewhat trivial example of a mixed state: it contains one filled LL of ${ }^{0} \mathrm{CFs}$ (composite fermions carrying zero vortices, i.e., electrons) and one filled LL of ${ }^{2} \mathrm{CFs}$. We denote this state by $\left(\nu^{(0)}, \nu^{(2)}\right)=(1,1)$, where $\nu^{(2 p)}$ is the filling factor of ${ }^{2 p}$ CFs. Upon attachment of two more vortices to each particle, a state at $4 / 11$ is obtained, which contains both ${ }^{2} \mathrm{CFs}$ and ${ }^{4} \mathrm{CFs}$, each at unit effective filling factor; in other words, 4/11 is described as $\left(\nu^{(2)}, \nu^{(4)}\right)=(1,1)$.

Wójs and Quinn [10 searched for a fully polarized FQHE at 4/11 numerically, through exact diagonalization on an $N=8$ particle system. They found no gap in the excitation spectrum here; as a matter of fact, the ground state here is not even uniform (it does not have $L=0$ in the spherical geometry, where $L$ is the total angular momentum). They concluded, based on this study, that there is no FQHE at 4/11, at least for fully polarized electrons. This result is not surprising in view of the fact that the fully polarized state at $4 / 3$ is rather fragile even for electrons, quite close to an instability [11], because the Coulomb matrix elements in the second Landau level are less repulsive than those in the lowest Landau level. The attachment of two further vortices to each electron to obtain the state at 4/11 would only further weaken it, most likely destabilizing it altogether.

In order to resolve the apparent discrepancy between 
theory and experiment, we consider a non-fully polarized FQHE state at 4/11. At least two such states are possible; our focus will be on the state in which both spin up ${ }^{4} \mathrm{CFs}$ and spin down ${ }^{2} \mathrm{CFs}$ fill one Landau level each: $\left(\nu_{\uparrow}^{(2)}, \nu_{\downarrow}^{(4)}\right)=(1,1)$. (Here, the subscript of $\nu$ refers to the spin of the composite fermion.) This state is related to $\left(\nu_{\uparrow}^{(0)}, \nu_{\downarrow}^{(0)}\right)=\left(1, \frac{1}{3}\right)$ as shown in Fig. 11. The ground state of this kind has been considered earlier by MacDonald in the context of generalized Laughlin states 12. It is the first member of the sequence

$$
f^{\prime}=\frac{1+f}{2(1+f) \pm 1}
$$

with $f$ given in Eq. (1). For the following reasons, we believe that $\left(\nu_{\uparrow}^{(2)}, \nu_{\downarrow}^{(4)}\right)=(1,1)$ will be a stable FQHE state at $4 / 11$ in an approprite range of Zeeman energies. First, an exact diagonalization study on sphere with $N=6$ electrons tells us that the ground state at $\nu=4 / 11$ is an $L=0$ state with partial polarization (to be specific, total spin quantum number is $S=1$ ) even with a very small Zeeman splitting energy [13]. Secondly, as we will see below, the wave functions of the composite fermion theory obtain not only the correct spin and angular momentum quantum numbers, but also accurate energies. Finally, and most importantly, higher electronic Landau levels are not used for the construction of this state, and the argument given above regarding the instability of the fully polarized 4/11 state is not effective here. The partially polarized state at $4 / 11$ is expected to be more robust than the fully polarized one for the same reason that the $1 / 3$ state in the second LL is rather weak but the $1 / 3$ state in the spin-reversed lowest LL is strong.

We will use the spherical geometry [14] below, which considers $N$ electrons on the surface of a sphere in the presence of a radial magnetic field emanating from a magnetic monopole of strength $Q$, which corresponds to a total flux of $2 Q \phi_{0}$ through the surface of the sphere. The wave function for the $\mathrm{CF}$ state at $Q$, denoted by $\Psi_{2 Q}$, is constructed by analogy to the wave function of the corresponding electron states at $q$, denoted by $\Phi_{2 q}$ :

$$
\Psi_{2 Q}=\mathcal{P}_{L L L} \Phi_{N-1}^{2 p} \Phi_{2 q}
$$

Here $\Phi_{N-1}=\prod_{j<k}\left(u_{j} v_{k}-u_{k} v_{j}\right)$ is the wave function of the fully occupied lowest Landau level with monopole strength equal to $(N-1) / 2$, where $u_{j} \equiv$ $\cos \left(\theta_{j} / 2\right) \exp \left(-i \phi_{j} / 2\right)$ and $v_{j} \equiv \sin \left(\theta_{j} / 2\right) \exp \left(i \phi_{j} / 2\right)$. $\mathcal{P}_{L L L}$ denotes projection of the wave function into the lowest Landau level (LLL). The monopole strengths for $\Phi_{2 q}$ and $\Psi_{2 Q}, q$ and $Q$, respectively, are related by $Q=q+p(N-1)$. For the ground state and the single exciton state, the wave functions $\Phi_{2 q}$ are completely determined by symmetry (i.e., by fixing the total orbital angular momentum $L$, which is preserved in going from $\Phi_{2 q}$ to $\Psi_{2 Q}$ according to the above rule), giving parameterfree wave functions $\Psi_{2 Q}$ for the ground and single-exciton states of interacting electrons. These have been found to be extremely accurate in tests against exact diagonalization results available for small systems [3, 4, 15].

To be concrete, we write a trial wave function for the state at $\nu^{*}=4 / 3$ as follows:

$$
\Phi_{\nu^{*}=4 / 3}^{g r}=\prod_{i, j \in \uparrow}\left(u_{i} v_{j}-v_{i} u_{j}\right) \prod_{k, l \in \downarrow}\left(u_{k} v_{l}-v_{k} u_{l}\right)^{3}
$$

where, for example, $i \in \uparrow$ denotes that the $i$-th particle is spin-up. Note that the spin part of the wave function is not explicitly written; the full wave function is obtained by multiplying the above wave function by the spin part and then antisymmetrizing the product. Upon the attachment of two vortices, the $\mathrm{CF}$ wave function for the ground state at $\nu=4 / 11$ is given by:

$$
\begin{aligned}
\Psi_{\nu=4 / 11}^{g r} & =\prod_{i, j \in \uparrow}\left(u_{i} v_{j}-v_{i} u_{j}\right)^{3} \prod_{k, l \in \downarrow}\left(u_{k} v_{l}-v_{k} u_{l}\right)^{5} \\
& \times \prod_{m \in \uparrow, n \in \downarrow}\left(u_{m} v_{n}-v_{m} u_{n}\right)^{2}
\end{aligned}
$$

Before proceeding further, let us make sure that $\Psi_{\nu=4 / 11}^{g r}$ is an eigenstate of the total spin, which can be shown as follows 12 . First, $\Psi_{\nu=4 / 11}^{g r}$ has the same totalspin eigenvalue as $\Phi_{\nu^{*}=4 / 3}^{g r}$ because $\Psi_{\nu=4 / 11}^{g r}$ is obtained by multiplying $\Phi_{\nu^{*}=4 / 3}^{g r}$ by a symmetric polynomial. Because the spin-up Landau level is full, application of the total spin raising operator annihilates $\Phi_{\nu^{*}=4 / 3}^{g r}$. Also, $\Phi_{\nu^{*}=4 / 3}^{g r}$ is evidently an eigenstate of $S_{z}$, and therefore is an eigenstate of total spin with $S=S_{z}=\left(N_{\uparrow}-N_{\downarrow}\right) \hbar / 2$, where $N_{\uparrow}$ and $N_{\downarrow}$ are the number of spin-up and spindown electrons, respectively. This argument is valid for any state that has all single-particle orbitals of one spin fully occupied.

Having established that $\Psi_{\nu=4 / 11}^{g r}$ is a legitimate wave function, we turn to the problem of energetics. Fig. 2 shows $N$-dependence of the energy of the ground state wave function described by Eq. (5). The pure Coulomb interaction $V(r)=e^{2} / \epsilon r$ is assumed here and below. By using the linear extrapolation, the ground state energy is estimated to be $-0.420527(14)$ in units of $e^{2} / \epsilon l_{0}$ in the thermodynamic limit. Here $l_{0}$ is the magnetic length at $\nu=4 / 11$ and $\epsilon$ is the dielectric constant of the background material. It is quite comparable to the energies of the fully polarized states at $1 / 3$ and $2 / 5$ [15].

In order to test the stability of this state, we consider its neutral and charged excitations. If it is found that an "excitation" has lower energy than the presumed ground state, we clearly have a wrong "ground state". While this procedure obviously cannot capture every possible instability, it has proven to be extraordinarily powerful in the past in ruling out FQHE states at low filling factors as well as in higher Landau levels [16].

The wave functions for the lowest-lying excitations are constructed by promoting a ${ }^{4} \mathrm{CF}$ into its lowest unoc- 
cupied ${ }^{4} \mathrm{CF}$-LL, while preserving its spin. Making an excitation in the ${ }^{2} \mathrm{CF}$ part will produce a higher energy excitation for the same reason as the excitation gaps are larger at $n /(2 n+1)$ than at $n /(4 n+1)$. Therefore, the wave function for excitations is written as follows:

$$
\begin{aligned}
\Psi_{\nu=4 / 11}^{e x}(L) & =\prod_{m \in \uparrow, n \in \downarrow}\left(u_{m} v_{n}-v_{m} u_{n}\right)^{2}>\prod_{i, j \in \uparrow}\left(u_{i} v_{j}-v_{i} u_{j}\right)^{3} \\
& \times \mathcal{P}_{L L L}\left[\prod_{k, l \in \downarrow}\left(u_{k} v_{l}-v_{k} u_{l}\right)^{4} \operatorname{Det}\left[\Phi_{2 q^{*}, \downarrow}^{e x}(L)\right]\right]
\end{aligned}
$$

where $L$ is the total angular momentum and $2 q^{*}=N_{\downarrow}-1$. The number of spin-up electrons is related to that of spindown electrons: $N_{\uparrow}=3 N_{\downarrow}-2$. Of course, if $\Phi_{2 q^{*}, \downarrow}^{e x}$ is replaced by the ground-state wave function at $q^{*}$, $\Phi_{2 q^{*}, \downarrow}^{g r}=\prod_{k, l \in \downarrow}\left(u_{k} v_{l}-v_{k} u_{l}\right)$, Eq. (5) is obtained. Comparison with exact diagonalization studies sheds light on the accuracy of the above wave functions. For $N=6$ system, the energies of the ground and excited state are approximately $0.2 \%$ larger than the exact energies; for $N=6$ and $Q=6.5$, the energies from the wave functions are $-0.473953(14)$ and $-0.473611(16) e^{2} / \epsilon l_{0}$ for the ground and the excited states, respectively, which are to be compared to the exact energies -0.4751 and -0.4742 $e^{2} / \epsilon l_{0}[13]$.

The energy gap of the lowest-lying excitations,

$$
\begin{aligned}
\Delta(k) & =\frac{\left\langle\Phi_{\nu=4 / 11}^{e x}(L)|V(r)| \Phi_{\nu=4 / 11}^{e x}(L)\right\rangle}{\left\langle\Phi_{\nu=4 / 11}^{e x}(L) \mid \Phi_{\nu=4 / 11}^{e x}(L)\right\rangle} \\
& -\frac{\left\langle\Phi_{\nu=4 / 11}^{g r}|V(r)| \Phi_{\nu=4 / 11}^{g r}\right\rangle}{\left\langle\Phi_{\nu=4 / 11}^{g r} \mid \Phi_{\nu=4 / 11}^{g r}\right\rangle},
\end{aligned}
$$

is computed using Monte Carlo methods in the spherical geometry. One of the most challenging aspects of the computation stems from the fact that the gap at $\nu=4 / 11$ is extremely small throughout the whole dispersion of the excitation. In fact, it is the smallest gap ever calculated in the quantum Hall effect; it is roughly 50 times smaller than the gap at $\nu=1 / 3$. As a result, the number of iterations of the Monte Carlo simulation must be increased significantly in order to minimize the statistical error, making the computations tremendously more time-consuming than for the primary states. Typically, 100 million Monte Carlo iterations were needed for each energy to obtain the desired accuracy, which is an order or magnitude larger than the number of iterations used in the studies of primary states ( $\sim 10$ million). Another consequence of the smallness of the gaps is that the intrinsic error in the gaps is not negligible. A comparison with exact diagonalization studies (for 6 particles) shows that even though the energies of the ground and excited state are predicted correctly at the level of $0.2 \%$, the gaps are reliable only to $10 \%$. Such an error is acceptable in view of the significant Monte Carlo uncertainty as well as our neglect of a number of other effects that make much bigger corrections.

Fig. 3 shows the dispersion curve of the lowest excitation. The results are plotted as a function of the wave vector of the excitation, $k$, which is related to the angular momentum $L$ via $k=L / R$ with $R$ being the radius of the sphere. The transport gap, which is the large wave vector limit of the dispersion curve, is estimated to be $0.002(1) e^{2} / \epsilon l_{0}$. Two roton minima are predicted in the (6ylispersion near $k l_{0}=0.7$ and 1.3 with energies of around $0.001 e^{2} / \epsilon l_{0}$. While the full dispersion is in principle observable in Raman scattering, the rotons may be easier to detect 17] The above numbers ought to be taken as no more than rough estimates of the actual experimental gaps because of the neglect in our calculation of various realistic effects such as finite transverse thickness, Landau level mixing and disorder. Previous studies on the effects of finite thickness and Landau level mixing 18 give 30 to $50 \%$ reduction of the gap. Therefore the estimated gap at $\nu=4 / 11$ is even smaller than the gap at $\nu=5 / 2$ [8], whose Hall plateau is firmly established only at ultra low temperatures $\sim 4 \mathrm{mK}[6]$.

We end with a few comments. First, a spin-singlet state at 4/11 can also be constructed, starting from the spin-singlet state at $4 / 3$ [19]. It is likely that it has lower energy than the one considered above at very small Zeeman energies, but it is not possible to obtain reliable quantitative information for this state due to the technical difficulties arising from the fact that they involve inverse flux attachment. Second, a clear message of our work is that the 4/11 FQHE is not fully polarized, which ought to be testable in tilted field experiments. Because the experiments do observe a minimum at rather high magnetic fields, we suspect that the actual observed state might be the partially spin polarized rather than spinsinglet. Finally, it is straightforward to enumerate other states that will exhibit FQHE at the non-principal fractions; the ones that are strongest are those that do not involve higher electronic Landau levels in their construction (at intermediate steps - of course, all states are eventually projected into the lowest electronic Landau level).

¿This work was supported in part by the National Science Foundation under Grant No. DMR-9986806. We thank V.W. Scarola for numerous helpful discussions, W. Pan for sharing his data prior to publication, and the Numerically Intensive Computing Group led by V. Agarwala, J. Holmes, and J. Nucciarone, at the Penn State University CAC for assistance and computing time with the LION-X cluster.

$\dagger$ Present address: Department of Physics, Sloane Physics Laboratories, Yale University, New Haven, CT 06520 
[1] D.C. Tsui, H.L. Stormer, and A.C. Gossard, Phys Rev. Lett. 48, 1559 (1982).

[2] J.K. Jain, Phys. Rev. Lett. 63, 199 (1989); Phys. Rev. B 41, 7653 (1990); Physics Today 53, no. 4, 39 (2000).

[3] Composite Fermions, edited by Olle Heinonen (World Scientific, New York, 1998).

[4] Perspectives in Quantum Hall Effects, edited by S. Das Sarma and A. Pinczuk (Wiley, New York, 1997).

[5] R.L. Willett et al., Phys. Rev. Lett. 59, 1779 (1987).

[6] W. Pan et al., Phys. Rev. Lett. 83, 3530 (1999); Physica E 6, 14 (2000).

[7] M. Greiter, X.G. Wen, F. Wilczek, Phys. Rev. Lett. 66, 3205 (1991); Nucl. Phys. B 374, 567 (1992); G. Moore and N. Read, Nucl. Phys. B 360, 362 (1991); K. Park, V. Melik-Alaverdian, N.E. Bonesteel, and J.K. Jain, Phys. Rev. B 58, R10167 (1998).

[8] V.W. Scarola, K. Park, and J.K. Jain, Nature 406, 863 (2000).

[9] The plateau at $\nu=4 / 11$ has not been fully established, but there is a reasonably well developed minimum of $R_{x x}$ at $\nu=4 / 11$; H.L. Stormer, Bulletin of the American Physical Society 45, 643 (2000); W. Pan, private communication.

[10] A. Wójs and J.J. Quinn, Phys. Rev. B 61, 2846 (2000); the same exact diagonalization study has been reported in the chapter by J.K. Jain and R.K. Kamilla in Ref. [3].

[11] T. Sbeouelji et al., Phys. Rev. B, in press (2000); V.W. Scarola, K. Park and J.K. Jain, unpublished.

[12] A.H. MacDonald, Surf. Sci. 229, 1 (1990).

[13] X.G. Wu and J.K. Jain, Phys. Rev. B 49, 7515 (1994).

[14] For the details about spherical geometry and monopole harmonics, see T.T. Wu and C.N. Yang, Nucl. Phys. B 107, 365 (1976); F.D.M. Haldane, Phys. Rev. Lett. 51, 605 (1983).

[15] J.K. Jain and R.K. Kamilla, Int. J. Mod. Phys. B 11, 2621 (1997); Phys. Rev. B 55, R4895 (1997).

[16] R.K. Kamilla and J.K. Jain, Phys. Rev. B 55, R13417 (1997); V.W. Scarola, K. Park, and J.K. Jain, preprint.

[17] A. Pinczuk et al., Phys. Rev. Lett. 70, 3983 (1993); Surf. Sci. 229, 384 (1990); Moonsoo Kang et al., to be published; H.D.M. Davies et al., Phys. Rev. Lett. 78, 4095 (1997); C.J. Mellor et al, Phys. Rev. Lett. 74, 2339 (1995); U. Zeitler et al., Phys. Rev. Lett. 82, 5333 (1999).

[18] F.C. Zhang and S. Das Sarma, Phys. Rev. B. 33, 2903 (1986); R. Price and S. Das Sarma, Phys. Rev. B 52, 17032 (1995); V. Melik-Alaverdian and N. Bonesteel, Phys. Rev. Lett. 79, 5286 (1997); V.W. Scarola, K. Park, and J.K. Jain, Phys. Rev. B 61, 13064 (2000).

[19] X.G. Wu, G. Dev, and J.K. Jain, Phys. Rev. Lett. 71, 153 (1993).
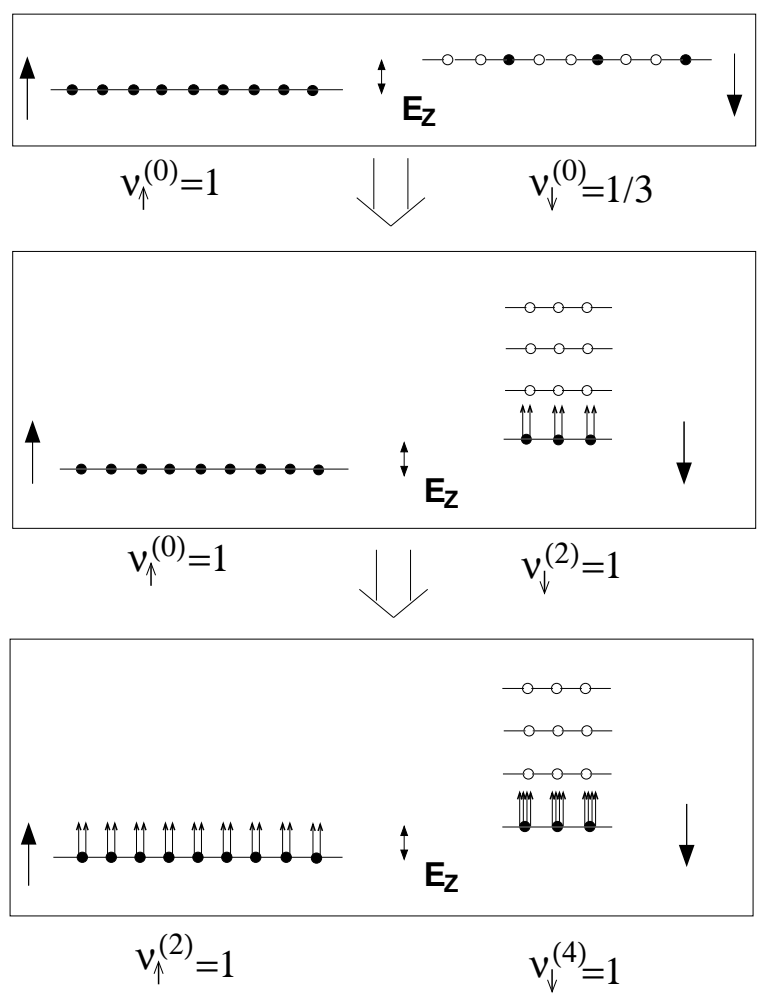

FIG. 1. Schematic diagram explaining the physics of the mixed CF state at $\nu=4 / 11$. Small arrows decorating the circles depict the vortices captured by composite fermions. Empty circles indicate empty sites in a given Landau level. Big arrows near the Landau levels signify the spin of the composite fermions. The spin-up and spin-down Landau levels are shifted in energy by the Zeeman splitting energy $E_{z}$. The top panel shows electrons at $\nu=4 / 3=1+1 / 3$ with the spin up Landau level fully occupied and the spin down Landau level one third occupied. The middle panel shows that the partially filled LL splits into Landau levels of composite fermions, with $1 / 3$ filling corresponding to unit filling of ${ }^{2} \mathrm{CFs}$. Attachment of two vortices to each particle produces the partially polarized 4/11 state studied in this article (bottom panel), which contains one filled ${ }^{4} \mathrm{CF}-\mathrm{LL}$ and one filled ${ }^{2} \mathrm{CF}-\mathrm{LL}$, with two types of composite fermions carrying opposite spins. The filling factor of ${ }^{2 p} \mathrm{CFs}$ is denoted by $\nu^{(2 p)}$. 


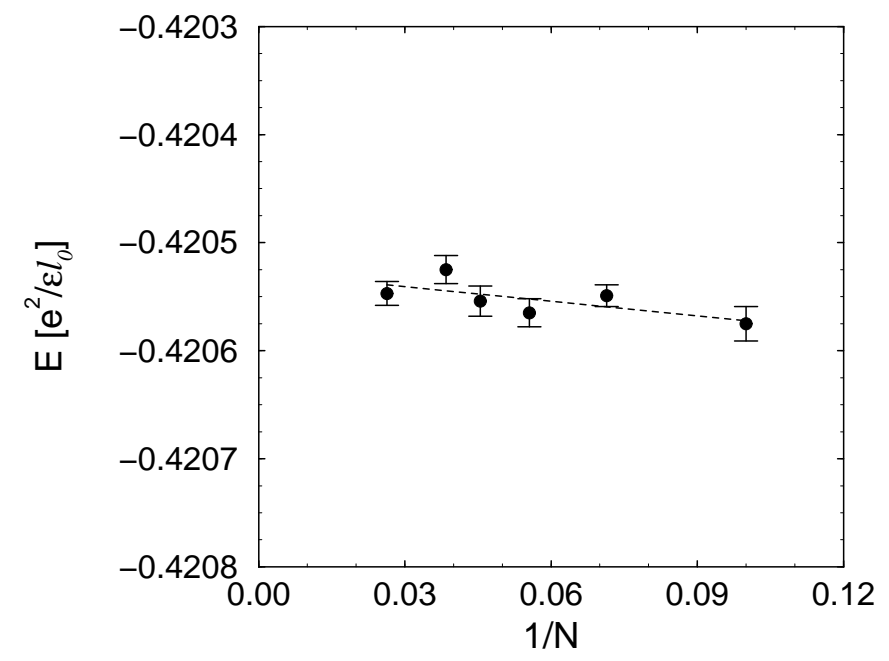

FIG. 2. Ground state energy at $\nu=4 / 11$ as a function of $N^{-1}$, the number of electrons. The quantity $l_{0}=\sqrt{\hbar c / e B}$ is the magnetic length, and $\epsilon$ is the dielectric constant of the background material. The error bars show one standard deviation in the Monte Carlo simulation.

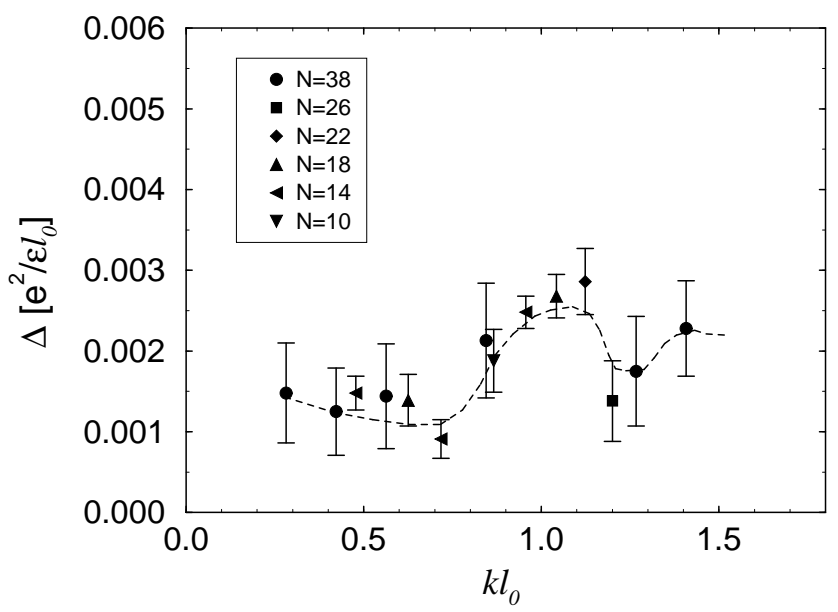

FIG. 3. Dispersion curve for the lowest excitations of the partially polarized FQHE state at $\nu=4 / 11$. Several values of $N$ are used to determine the entire curve. The dashed line is a guide to the eye. 